\title{
Using computer simulation to improve patient flow at an outpatient internal medicine department
}

Ortíz Barrios, Miguel Angel; López Meza, Pedro

\begin{abstract}
Objectives: The objective is to analyze the foundation the Virtual Network Level of Application Composed IP Networks Connected with Systems. Methods/Statistical Analysis: In this research using the methodological foundation logical analysis attempts to give an overview of the state of the art in terms P2P and especially networks in the field of computer systems distributed along with several contributions that allow build systems High Throughput Computing. Findings: The network is virtual is application level made up connected to IP networks, in a nutshell systems P2P systems are distributed systems in which there are no any central control or hierarchical structure and can therefore go beyond the services offered by traditional centralized type systems Client Server at the cost of greater technical and theoretical complexity. In P2P systems, nodes that make up the system are connected together using IP networks forming a kind of virtual network application layer, also called "Overlay", which is used to route messages between nodes in order to find information. Application/Improvements: The recent emergence of applications that exploit the network concept Peer to-Peer or P2P in which all members have the same role has made consider its possible applications to the world of distributed computing. Although there are already similar in this field, such as those that follow the paradigm of grid computing, this new concept may be the source systems massive computer wide open to anyone with an Internet connection could use for the benefit or for solutions others, sharing their surpluses recourses.
\end{abstract}

\section{Keywords}

Massive Computing Systems, Sis Issues Connected to IP Networks, Technical Complexity, Virtual Application Level 